\title{
STATISTICAL ISSUES IN DARK MATTER DETECTION
}

\author{
D.R. Tovey ${ }^{a}$, N.J.T. Smith ${ }^{b}$ \\ ${ }^{a}$ Department of Physics and Astronomy, University of Sheffield, Hounsfield Rd., Sheffield S3 7RH, UK. \\ ${ }^{b}$ Particle Physics Department, Rutherford Appleton Laboratory, Chilton, Didcot, Oxon OX11 0QX, UK.
}

\begin{abstract}
Statistical techniques used to analyse data from direct detection dark matter experiments are reviewed with an emphasis on general issues which could benefit from further study. In order to illustrate these issues three case studies are presented of detectors operating in different statistical regimes which require novel approaches to data analysis.
\end{abstract}

\section{INTRODUCTION}

Studies of stellar and galactic dynamics on progressively larger scales indicate the presence of large quantities of invisible or 'dark' matter which neither absorbs nor emits electromagnetic radiation [1]. This dark matter is invisible to conventional astronomy and yet makes up at least $90 \%$ of the mass density of the universe. It is now believed that the total mass density is $\sim 30 \%$ of the critical density [2] but the measured primordial abundances of the light elements are only found to be consistent with the predictions of Big Bang Nucleosynthesis (BBN) for a baryonic contribution to this density $\lesssim 5 \%$. This indicates that the majority of the dark matter is non-baryonic in form. Stable particle candidates for this non-baryonic dark matter are provided by many theories extending the Standard Model, however the leading contender is generally the Lightest Supersymmetric Particle (LSP) from R-Parity conserving SUSY theories [1]. The LSP (typically the lightest neutralino) is just one of a broad class of candidates collectively referred to as Weakly Interacting Massive Particles (WIMPs). Any positive evidence for the existence of WIMP dark matter would have profound implications for both cosmology and particle physics.

\section{DIRECT DETECTION EXPERIMENTS}

Models which predict the existence of WIMP dark matter such as supersymmetry typically also predict that WIMPs couple weakly to baryonic matter such as atomic nuclei [3]. Studies of the dynamics of stars within spiral galaxies such as our own also indicate that a relatively large amount of the dark matter is trapped within the potential wells of these galaxies [1]. It may therefore be possible to detect WIMP dark matter by searching for the elastic scattering of nuclei contained in earth-borne detectors. The observed energy spectrum $d R / d E_{v}$ of such nuclear recoils depends upon a number of factors related to the kinematics of the scattering process and the phase- space distribution of WIMPs trapped in the galaxy ( $S\left(A, E_{R}\right)$ where $A$ is the target atomic mass and $E_{R}$ is the nuclear recoil energy), the nuclear physics of the mass and spin distributions of the nuclei (form-factors $F^{2}\left(A, E_{R}\right)$ and coupling enhancements $I(A)$ ) and the particle physics of the WIMPs themselves (mass and WIMP-nucleon interaction cross-section $\left.\sigma_{p}\right)$. Other detector dependent factors include the mass fraction of element $A$ in the target $(f(A))$, the nuclear recoil quenching factor $\left(g(A)\right.$ - defined below) and the detector energy response matrix $\left(\epsilon\left(E_{v}\right)\right)$. The resulting energy spectrum formula is:

$$
\frac{d R}{d E_{v}}=\sigma_{p} \Sigma_{A} f(A) \cdot S\left(A, E_{R}\right) \cdot I(A) \cdot F^{2}\left(A, E_{R}\right) \cdot g(A) \cdot \epsilon\left(E_{v}\right)
$$

Of the terms appearing in the above formula the kinematic factor and nuclear form-factors lead to an overall recoil spectrum which falls rapidly with increasing energy (Fig. 1) thus making the use of detectors with low recoil energy thresholds $(\lesssim 10 \mathrm{keV})$ especially important. The coupling enhancements 
lead to increased scattering cross-sections for heavy nuclei (scalar interactions) and those with large spin (axial-vector interactions) through nuclear coherence effects. This further constrains the choice of detector materials. The final consideration is that the expected WIMP interaction rate is extremely small ( $\lesssim 1 / \mathrm{kg} /$ day) and in particular it is several orders of magnitude smaller than that of the main source of background events, namely electron recoils caused by beta decay and Compton scattering of naturally occurring background radiation. This background event rate must therefore be reduced, initially by shielding detectors with high purity, high- $Z$ materials and by purifying detector components to remove radioactive contaminants such as isotopes from the $\mathrm{U}$ and $\mathrm{Th}$ decay chains. Operation of experiments deep underground is important for removing background nuclear recoils caused by elastic scattering of high energy neutrons from cosmic ray spallation but also reduces the ambient level of photon radiation.
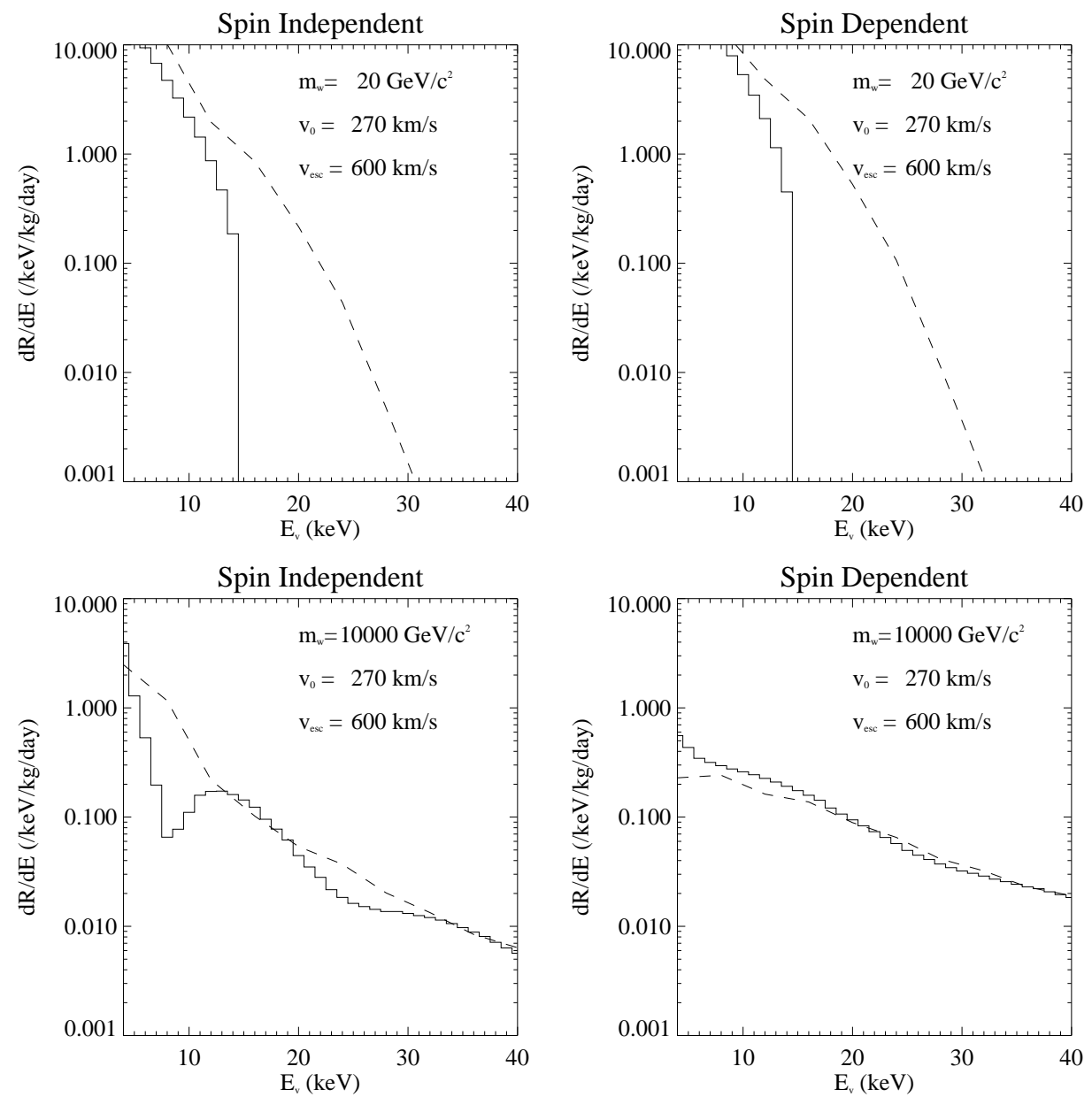

Fig. 1: Simulated WIMP induced nuclear recoil spectra in a NaI(Tl) scintillator detector. The plots correspond to spectra expected for $20 \mathrm{GeV} / \mathrm{c}^{2}$ (top) and $10 \mathrm{TeV} / \mathrm{c}^{2}$ (bottom) WIMPs, for spin independent (left) and spin dependent (right) WIMPnucleon interactions. The full histograms are raw spectra obtained from Eqn. (1) without taking into account detector response while the dashed lines show the same spectra following convolution with the detector response matrix [4].

A further measure which can be taken to reduce the rate of background events is to use a detector material possessing electron recoil discrimination properties. Such properties generally derive from the differing $d E / d x$ (energy loss) of nuclei and electrons of similar recoil energies, which can lead to increased 'quenching' of charge produced in solid and liquid targets. Examples include scintillation 
pulse-shape differences in materials such as $\mathrm{NaI}(\mathrm{Tl})$ [5] and liquid xenon [6], reduced electron to photon ratios in double-phase xenon detectors [7], reduced photon to phonon ratios in cryogenic scintillation detectors [8] and reduced charge to phonon ratios in cryogenic ionisation detectors [9]. It is important to note however that in addition to reducing the rate of background events the use of such techniques permits detectors to actively discover a WIMP dark matter signal rather than just set limits on its interaction rate or cross-section. The reason for this is that direct detection experiments, unlike collider experiments, operate in a regime where the absolute rate and energy spectrum of background events is only poorly known. This means that it is difficult to identify a signal purely as an excess of events above expected background, as is the case with a counting experiment. Through the use of a discriminating variable measuring one of the discriminating properties listed above however, the background rate in the signal region can be determined through a combination of signal and background calibration and measurement of the event rate in the background dominated region. Detector sensitivity can be improved significantly in this way and the use of detector materials possessing intrinsic background discrimination properties is now the main factor influencing the design of direct detection experiments.

\section{ANALYSING DATA}

The need to make optimum use of all information provided by a detector drives the choice of statistical data analysis techniques. In smaller detectors $(\lesssim 50 \mathrm{~kg})$ this information is generally confined to a measurement of event energy and one discriminating parameter. At present the analysis typically proceeds in two steps:

1. Analyse the distribution of the discriminating parameter using a Gaussian fit or cut-based Poisson analysis to determine the number of nuclear recoil signal events contained within the data. No assumption is made about the origin of any nuclear recoils at this stage.

2. Interpret the measured number of nuclear recoil events in terms of a WIMP signal by fitting to the observed energy spectrum in order to define a confidence or exclusion region in WIMP mass/interaction cross-section parameter space.

This process has the advantage that the initial stage of the analysis is completely independent of the WIMP mass or WIMP-nucleon interaction cross-section and hence can be performed once for a given data set. All dependence on the WIMP parameters is 'factorised' out into the second stage, which in general is quicker to perform. An open question remains as to whether improved sensitivity can be obtained by combining these two steps into a single two-dimensional fit or event selection. If the WIMP model-independence of the discriminating parameter is total then there will presumably be little or no improvement however this has still to be confirmed.

In larger detectors ( $\gtrsim 50 \mathrm{~kg}$ ) additional information regarding the WIMP nature of a nuclear recoil signal can be provided by examining the time spectrum of candidate events. At different times of the year detectors move with different velocities relative to the 'halo' of dark matter particles trapped in our galaxy due to the earth's motion around the sun. This causes the average flux and hence nuclear recoil event rate to modulate by $\sim 3 \%$ over the course of the year. Searches for such an annual modulation signature are potentially fraught with systematic problems due to seasonal fluctuation of the background event rate, however the technique becomes competitive when using large detectors and several experiments have chosen to take this approach. The DAMA collaboration [10] in particular have claimed positive evidence for a signal using this technique (Fig. 2) and this is currently providing a focus for many of the experimental efforts around the world. An open question is whether annual modulation, background discrimination and energy information can be combined in a more efficient manner to improve detector sensitivity.

To illustrate the above considerations a number of case studies will now be considered. 


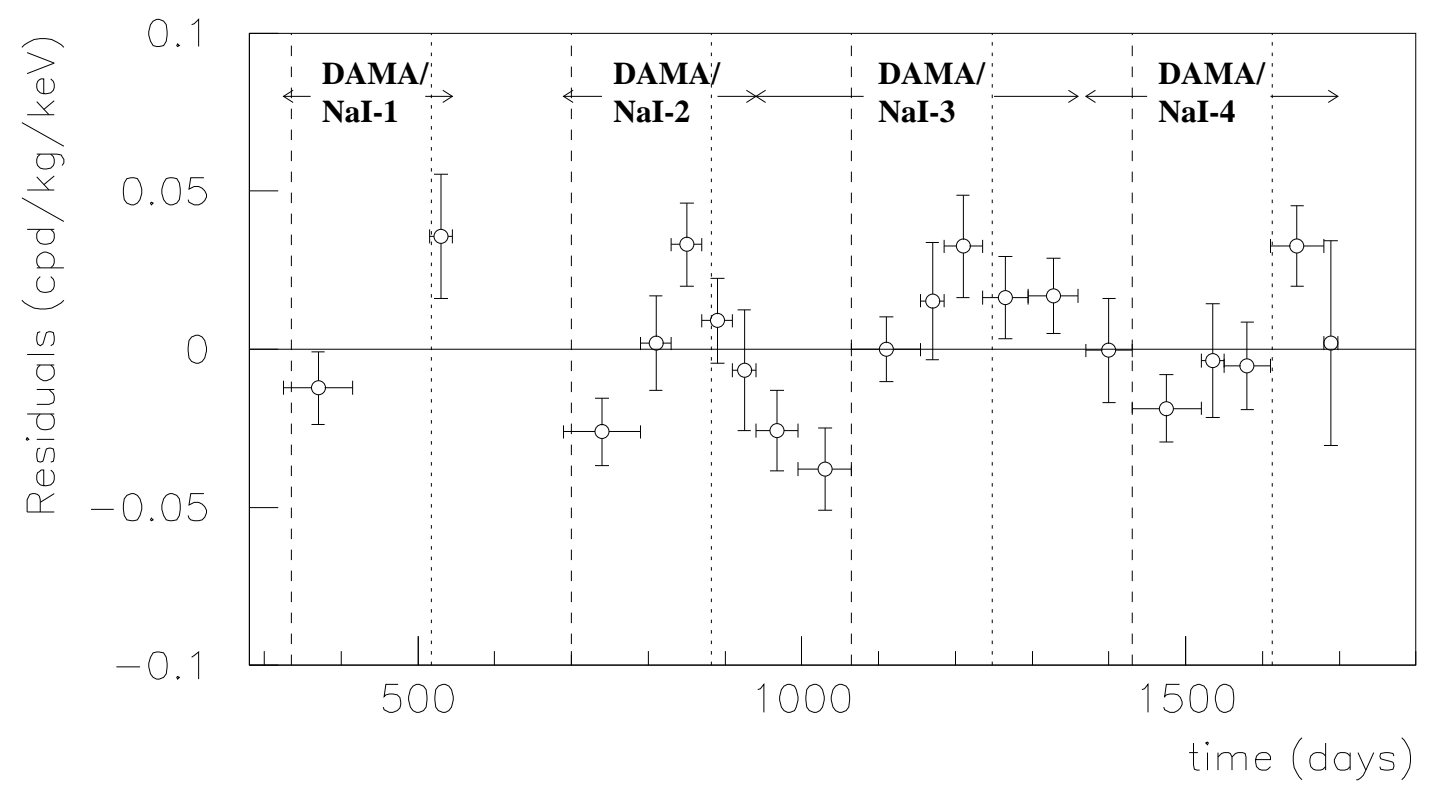

Fig. 2: Residual event rate following subtraction of the mean from the total event rate observed in the DAMA-NaI detector array as a function of time over the course of $57986 \mathrm{~kg}$.days of running [11].

\section{CASE STUDY 1: NAIAD}

The UK Dark Matter Collaboration [12] operates a $60 \mathrm{~kg}$ array of $\mathrm{NaI}(\mathrm{Tl})$ scintillator detectors ('NaIAD') at the Boulby Mine in North Yorkshire. Iodine is a heavy nucleus $(\mathrm{A}=127)$ with a large spin-independent coupling enhancement while both sodium and iodine possess significant spin-dependent coupling enhancements. $\mathrm{NaI}(\mathrm{Tl})$ is relatively radio-pure and offers a low recoil energy threshold due to its high light-output and favourable nuclear recoil quenching factors (the ratio of the numbers of photons emitted in nuclear and electron recoil events of the same recoil energy). A major additional advantage is that the time spectrum of emitted scintillation photons (the scintillation pulse-shape) depends sensitively on the type of recoil, permitting the use of Pulse-Shape Discrimination techniques (PSD). Simple PSD involves approximating the observed time spectrum with an exponential function and using the decay time constant of that function, determined using a $\chi^{2}$ fit or maximum likelihood estimator, as the required discriminating parameter. The background rejection efficiency provided by this technique for high signal efficiency is not sufficient to permit the use of a cut-based Poisson analysis (as in a counting experiment) and so instead a $\chi^{2}$ fit to the data with the sum of a calibrated (with a gamma source) electron recoil background distribution and an assumed nuclear recoil signal distribution is performed. In the absence of a significant population of signal events the fitted normalisation of the nuclear recoil distribution is found to be zero within errors. The magnitude of and error on this normalisation can then be interpreted in terms of a 90\% CL upper limit on the nuclear recoil signal rate using (in this case) a Bayesian prescription. The ensemble of these limits (as a function of energy) can then be used in Step 2 (above) to set limits on WIMP mass and interaction cross-section (Fig. 3). 


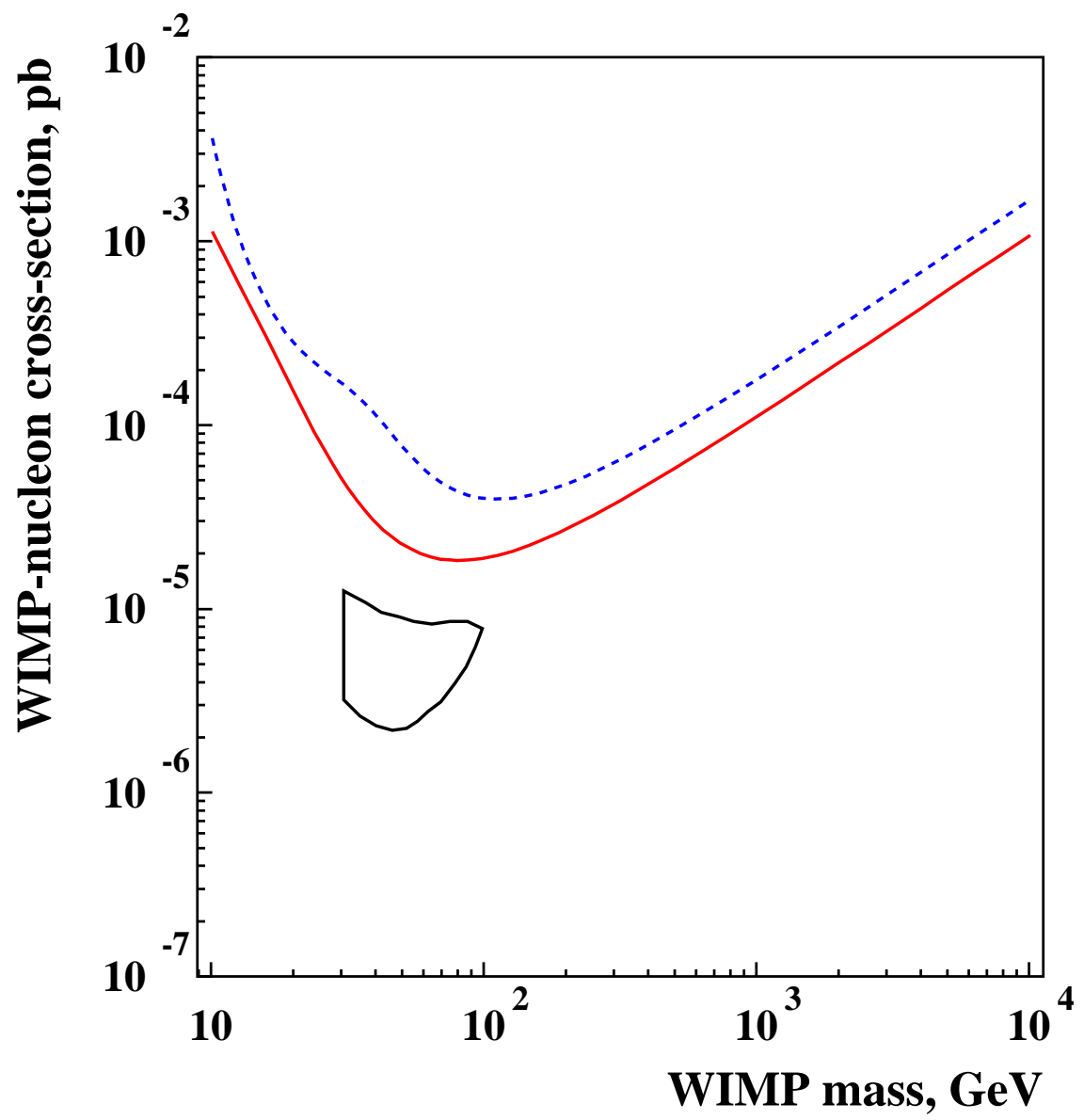

Fig. 3: 90\% CL upper limits on the WIMP-nucleon interaction cross-section for spin-independent interactions (full line) obtained from analysis of NaIAD data using techniques described in the text [13]. The dotted curve corresponds to earlier NaI(Tl) limits. The closed curve signifies the region favoured by the DAMA annual modulation analysis.

One open question relating directly to $\mathrm{NaI}(\mathrm{Tl})$ data arises from the fact that detailed analysis indicates that a gamma distribution rather than an exponential distribution provides the best approximation to the scintillation pulse-shape. Preliminary work suggests that the additional degrees of freedom allowed by this function do not provide any improvement in electron recoil discrimination efficiency, with, in particular, the finite rise-time of the pulse appearing to be independent of the type of recoil. Further study of this question is required however. Additional questions include whether it is possible to find an improved technique for estimating the decay time constant $\tau$ of the pulse in the presence of an unknown rate of noise photons, and whether the form of the distribution of decay time constants, which appears to be better approximated by a log-normal (Fig. 4) or inverse gamma distribution rather than the expected gamma distribution in $\tau$, can be explained theoretically. 

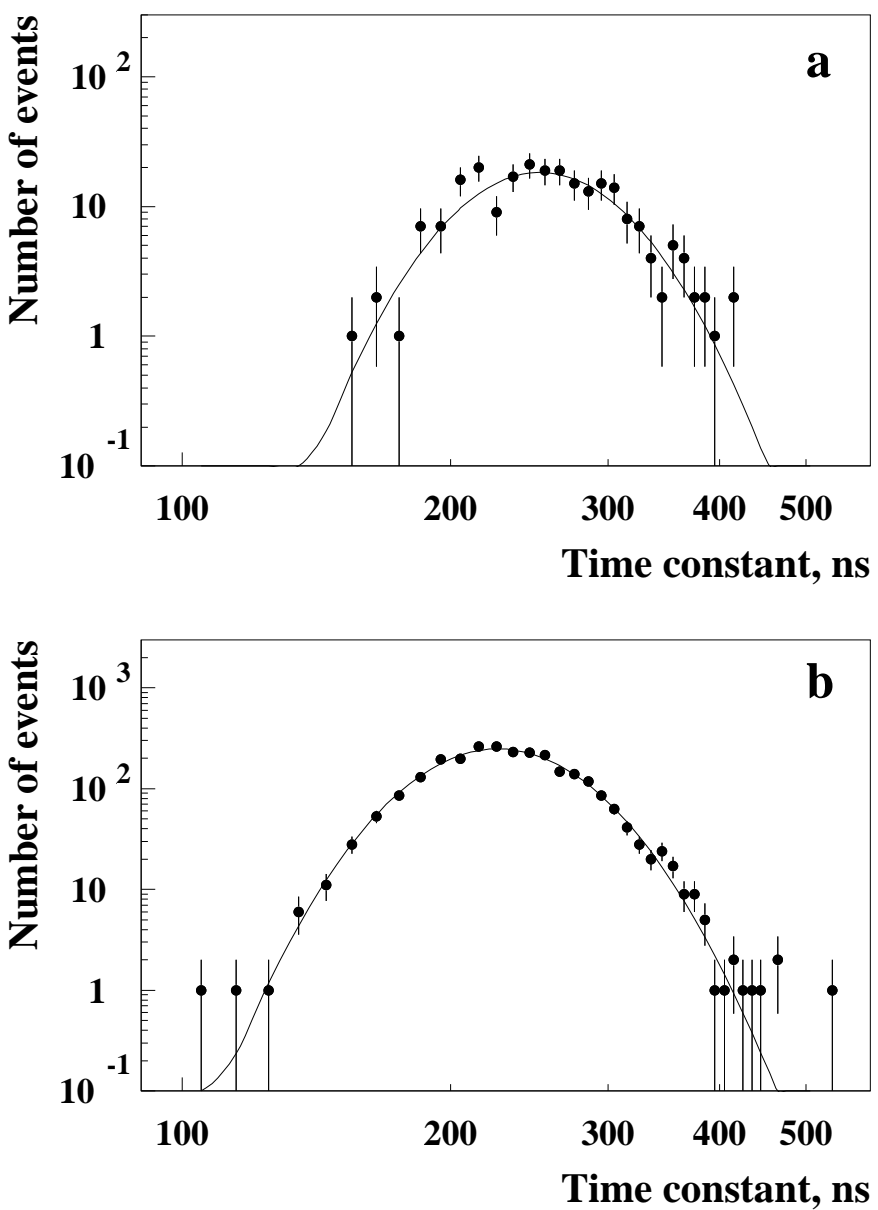

Fig. 4: Distribution of scintillation decay time constants estimated from NaIAD electron recoil calibration (a) and data run (b) data using techniques described in the text [13]. In Figure (a) the data are well fitted by a single log-normal distribution (solid curve), providing motivation for log-normal fit to the run data shown in Figure (b). These data are well fitted by a sum of two log-normal distributions (of variable width) corresponding to signal and background populations (solid curve). The fitted normalisation of the signal distribution is zero within errors indicating no evidence for signal events.

\section{CASE-STUDY 2: ZEPLIN 1}

The ZEPLIN I detector [14] is a single phase liquid xenon scintillation detector of $3 \mathrm{~kg}$ fiducial volume, viewed by three 3" quartz photomultipliers through silica windows and optically isolated, self shielding, liquid xenon turrets. The target is enclosed by a multi-purpose, 1 tonne, PXE-based liquid scintillator shield and an outer passive lead shield. The liquid scintillator shield acts as a veto for PMT events and also provides a Compton electron recoil calibration contemporaneous with the data collected, an active shield for external gammas, a high purity inner shield and, through the use of an optional internal gadolinium coated surface, a neutron monitor.

As in NaIAD, discrimination between nuclear recoil and electron recoil events is provided by the time constant of the scintillation light from the target [15]. For nuclear recoils initiated by neutrons or WIMPs the scintillation has a characteristic decay time approximately 55\% that of electron recoils above $10 \mathrm{keV}$ electron equivalent energy. The optimisation of the extraction of the nuclear recoil limit in Step 1 (above) using this information remains the key statistical question within the ZEPLIN I analysis. Several different estimation techniques have been applied to the scintillation pulses, including fitting routines 
based on single exponential fits, mean and median times. Although the time structure is well defined for large events, the events of interest are measured in tens of photo-electrons for which the model independent fits to the pulses provide similar results. The characteristic distribution of the scintillation time constant estimators is a gamma distribution in $1 / \tau$. As for NaIAD, the underlying process for the generation of this distribution in the photomultiplier signals is not well understood and needs further study.

Following time constant estimation, Step 1 of the analysis proceeds by comparison of the estimator distributions for the run data and electron recoil calibration data generated by gamma sources or known gamma backgrounds [16]. The latter is generated in ZEPLIN I through the use of the Compton veto signal where coincidental events in the chamber and veto are generated by high energy Compton scattered gammas from the photomultipliers. Should these estimator distributions have a relative shift in mean value, due either to the operating conditions of the target during calibrations or some spatial effect, then an appropriate normalisation must be applied to the calibration distributions. A $\chi^{2}$ analysis can then be used to compare the sum of electron and nuclear recoil calibration data with the run data to extract the 90\% CL upper limit on signal events. An alternative to the comparison with electron recoil calibration data is to assume that the estimator distributions of the scintillation data are in fact well represented by true gamma distributions or, less stringently, smooth monotonically rising curves. An analysis can then be performed by comparing the expected distribution for the sum of signal and background with the assumed functional form of the calibration data, or by searching for a gradient break in the monotonic distribution. The validity of this comparison with the assumed functional form of the calibration data is in question when the underlying process for the generation of such a distribution is unknown. The rigour of the statistical tests utilised to extract the $90 \%$ CL upper limit on the nuclear recoil rate is of key importance in present dark matter experiments due to the need to assess (in)compatability of crosssection limits (Fig. 5) with the DAMA signal region (above).

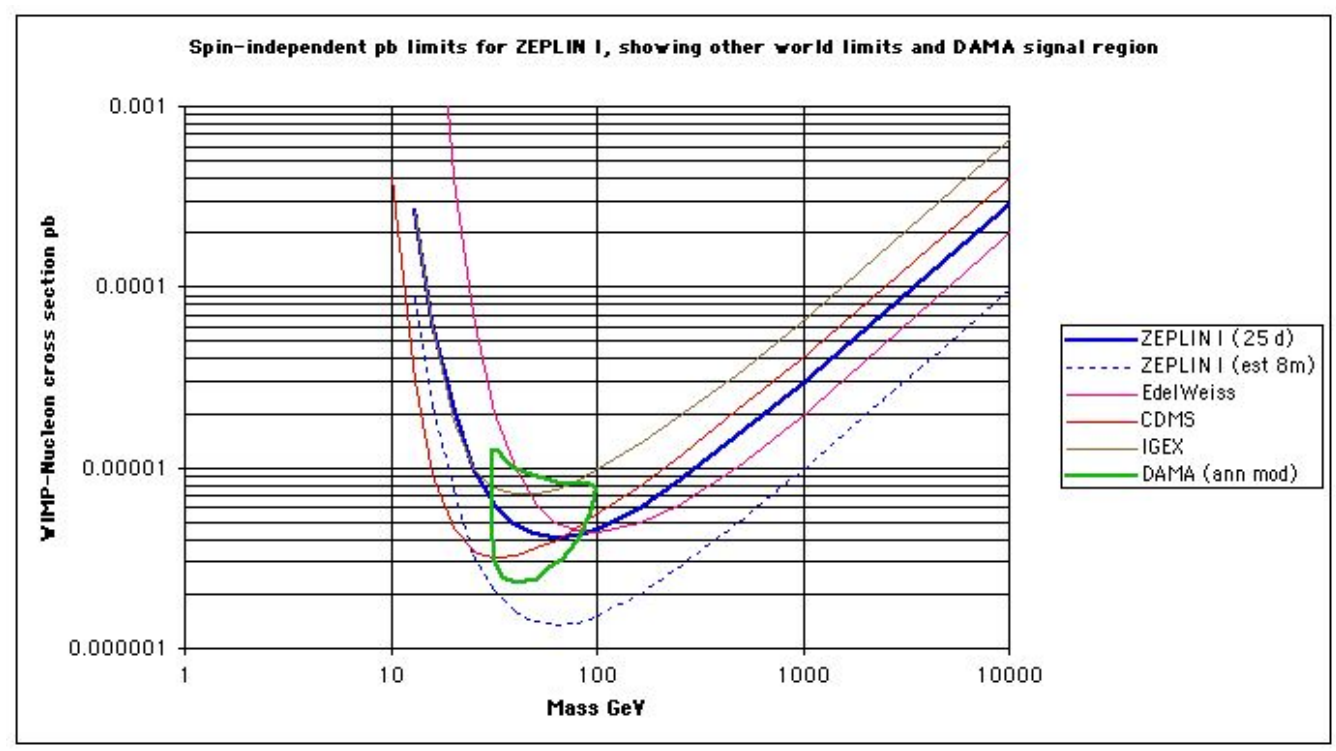

Fig. 5: The preliminary WIMP-nucleon interaction cross-section - WIMP mass limit set by ZEPLIN I from 25 days live-time compared to other direct dark matter detection experiments [17]. The full thick curve corresponds to the current ZEPLIN 1 90\% CL limit, while the dotted curve corresponds to the predicted sensitivity for eight months of data. The the three other curves (from the bottom on the right hand side) are equivalent $90 \%$ CL upper limits set by the EDELWEISS, CDMS and IGEX experiments respectively. The closed curve is the $4 \sigma$ allowed region claimed by the DAMA collaboration using the predicted annual modulation signature. 


\section{CASE-STUDY 3: DRIFT}

The UK Dark Matter Collaboration, together with LA Occidental and Temple Universities, is also developing another class of detector (DRIFT) in which target density has been reduced to enable unprecedented electron recoil discrimination efficiency to be obtained. A prototype detector (DRIFT-1) is currently operating at Boulby Mine and consists of a TPC containing $\sim 200 \mathrm{~g}$ of low pressure (40 Torr) $\mathrm{CS}_{2}$ gas read-out with two high granularity MWPCs [18]. Charge generated by recoil events attaches to the electronegative $\mathrm{CS}_{2}$ gas molecules forming $\mathrm{CS}_{2}^{-}$ions. The high mass of these ions limits transverse diffusion to $\lesssim 1 \mathrm{~mm}$ over drift distances in excess of $1 \mathrm{~m}$ and this, together with the excellent position resolution of the read-out plane, permits efficient discrimination between nuclear and electron recoils on the basis of measured track length. In addition to this benefit however, the technique allows the direction of candidate nuclear recoils to be determined. This is important because the rotation of the earth upon its axis causes the mean velocity relative to the detector of incident WIMPs, and hence WIMP-induced nuclear recoils, to modulate diurnally through $\sim 90^{\circ}$. Directional information can therefore be used to positively identify a potential nuclear recoil signal as being due to WIMP- scattering.

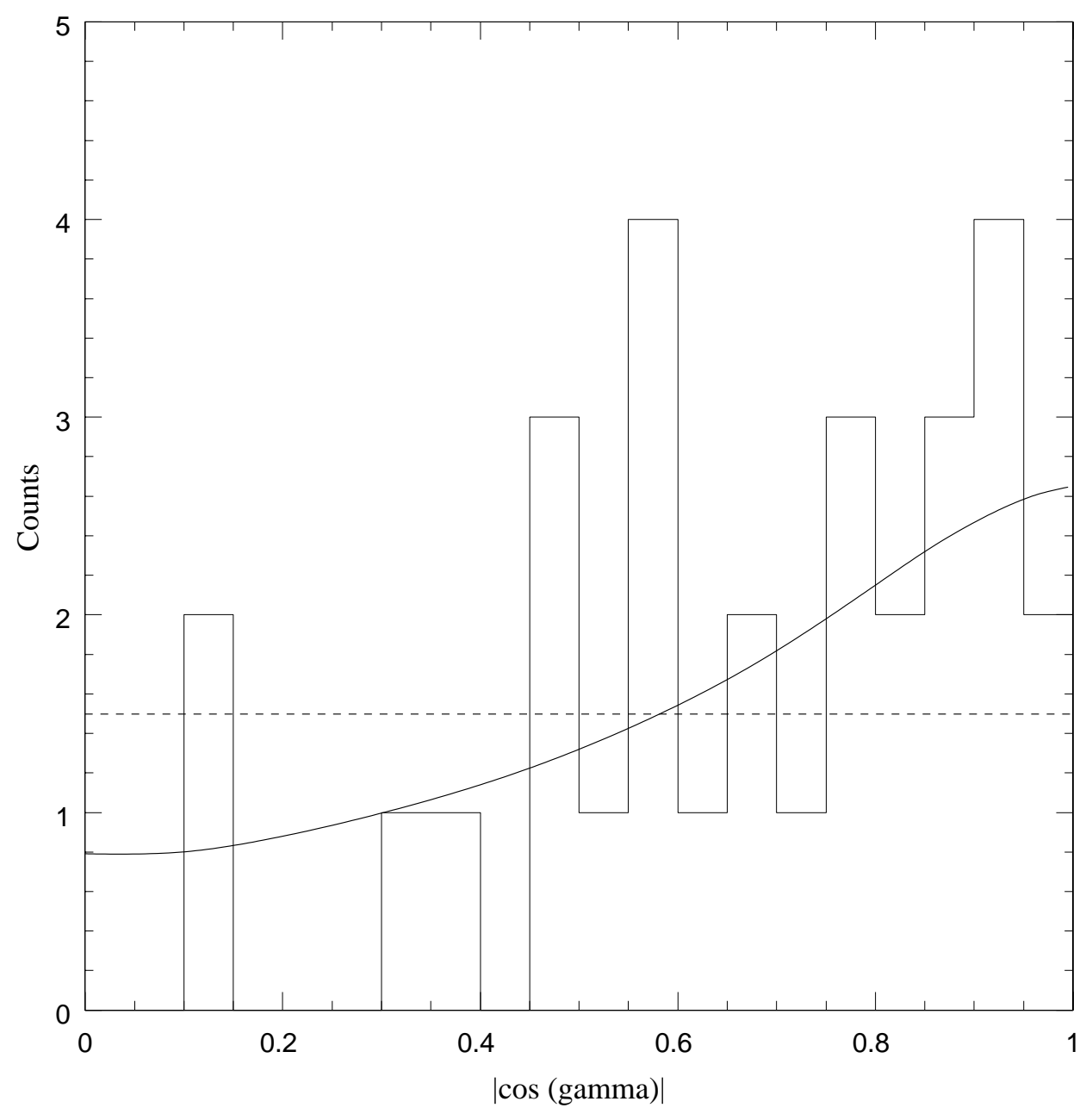

Fig. 6: Angular distribution of simulated nuclear recoil signal events in the DRIFT detector. $\cos (\gamma)=1$ corresponds to recoils pointing in the same direction as the mean expected WIMP flux vector. An anisotropy is evident at $90 \%$ CL (KS test statistic) for 30 signal events. Figure taken from [19]. 
At present it is intended that directional information will only be used once a signal has been seen, with, for instance, a Kolmogorov-Smirnov statistic being used to test whether the distribution in a suitable angular variable of events passing track length cuts is consistent with a uniform parent distribution characteristic of background (Fig. 6). An open question remains however regarding whether the information contained in this directional variable, together with track length and energy information can be used in a multi-variate analysis to significantly improve detector sensitivity. Such an analysis would undoubtedly be complicated by dependency of the directional discriminating parameters on the WIMP model, thereby preventing factorisation of the problem into two steps as discussed above. It is hoped however that demonstrable improvements may nevertheless be obtainable with the application of a suitable multi-variate technique.

\section{CONCLUSIONS}

Direct detection dark matter experiments offer unique challenges to experimentalists seeking to use advanced statistical techniques to advance our fundamental understanding of the universe. Beyond the usual issues relating to limit setting and the definition of rigorous confidence regions (which are common to many experiments) these experiments require novel approaches to parameter estimation from noisy data, multi-variate analyses in the presence of imperfectly known background distributions and many other techniques. It is hoped that a better understanding of at least some of these issues will lead to considerable improvements in detector sensitivity in the near future.

\section{ACKNOWLEDGMENTS}

The authors wish to acknowledge PPARC for providing financial support.

\section{References}

[1] G. Jungman, M. Kamionkowski, K.Griest, Phys. Rep. 267, 195 (1996).

[2] M.S. Turner, Proc. 2001: A Spacetime Odyssey, (U. Michigan, May 2001, World Scientific), astro-ph/0202008.

[3] M.W. Goodman, E. Witten, Phys. Rev. D31, 3059 (1985).

[4] D.R. Tovey, PhD Thesis, University of Sheffield, 1998 (unpublished).

[5] P.F. Smith et al., Phys. Lett. B379, 299 (1996).

[6] D. Akimov et al., Phys. Lett. B524, 245 (2002).

[7] D. Cline et al., Astropart. Phys. 12, 373 (2000).

[8] P. Di Stefano et al., Proc. 3rd Int. Workshop on the Identification of Dark Matter, 409 (World Scientific, 2001).

[9] R. Abusaidi et al., Phys. Rev. Lett. 84, 5699 (2000).

[10] R. Bernabei et al., Nucl. Phys. B91S, 361 (2001).

[11] R. Bernabei et al., Proc. DARK2002, (Cape Town, South Africa, 4-9 Feb 2002), to be published.

[12] N.J.C. Spooner et al., Phys. Lett. B473, 330 (2000).

[13] M. Carson et al., Proc. 5th Int. Symposium on Sources and Detection of Dark Matter in the Universe, (Marina del Rey, CA, February 2002), to be published. 
[14] I. Ivaniouchenkov et al., Proc. IEEE 2000 Nuclear Science Symposium and Medical Imaging Conference.

[15] G. J. Davies et al., Phys. Lett. B320, 395 (1994).

[16] N. J. T. Smith, C. H. Lally, G. J. Davies, Nucl. Phys. B48 (Proc. Suppl.), 67 (1996).

[17] References summarised at http://dendera.Berkeley.EDU/plotter/entryform.html

[18] C.J. Martoff et al., Proc. 2nd Int. Workshop on the Identification of Dark Matter, 389 (World Scientific, 1999).

[19] M.J. Lehner et al., Proc. Dark Matter in Astro- and Particle Physics, 590 (World Scientific, 2001). 